Published in final edited form as:

Ann Allergy Asthma Immunol. 2019 May ; 122(5): 532-533.e3. doi:10.1016/j.anai.2019.02.022.

\title{
Subcutaneous immunotherapy in patients with eosinophilic esophagitis
}

\author{
Benjamin S. Robey, MD¹, Swathi Eluri, MD, MSCR ${ }^{1}$, Craig C. Reed, MD¹, Maya R. Jerath, \\ MD, PhD², Michelle L. Hernandez, MD², Scott P. Commins, MD², and Evan S. Dellon, MD, \\ $\mathrm{MPH}^{1}$ \\ ${ }^{1}$ Center for Esophageal Diseases and Swallowing, and Center for Gastrointestinal Biology and \\ Disease, Division of Gastroenterology and Hepatology; \\ ${ }^{2}$ Division of Allergy and Immunology; University of North Carolina School of Medicine, Chapel Hill, \\ NC
}

\section{Keywords}

Eosinophilic Esophagitis; Subcutaneous Immunotherapy; Oral Immunotherapy; Allergy; Immunology

\begin{abstract}
While the association between eosinophilic esophagitis (EoE) and food allergens is well established, a large proportion of EoE patients have concomitant atopic disease and there is growing evidence that aeroallergens may play a role in pathogenesis. ${ }^{1-3}$ However, subcutaneous immunotherapy (SCIT) for treatment of EoE has not been extensively described. ${ }^{4-8}$ The aim of this study was to compare characteristics and treatment outcomes in patients with EoE who were treated with SCIT (EoE+SCIT) to those who were not treated with SCIT (EoE-SCIT).
\end{abstract}

We conducted a retrospective cohort study using the University of North Carolina (UNC) EoE clinicopathologic database, ${ }^{9}$ focusing on patients of all ages from 2008 (the year that International Classification of Disease, version-9 [ICD-9] released an EoE code $)^{10}$ through 2016. To be included, patients met consensus guidelines for an incident diagnosis of EoE as published at the time of this study design. ${ }^{1}$ This study was approved by the UNC Institutional Review Board.

To capture all EoE patients as well as all patients treated with SCIT, we queried the Carolina Data Warehouse for Health, a central data repository containing granular patient information with searchable fields derived from the UNC electronic medical record. We used ICD-9/10 codes, as well as Current Procedure Terminology (CPT) codes for the search. EoE was identified with 530.13 and K20.0. SCIT treatments were identified with CPT codes 95115 ,

Corresponding author: Evan Dellon, MD, MPH, CB \#7080, Bioinformatics Building, 130 Mason Farm Rd., UNC-CH, Chapel Hill, NC 27599-7080, Phone: (919) 966-2513, Fax:(919) 843-2508, edellon@ med.unc.edu.

Publisher's Disclaimer: This is a PDF file of an unedited manuscript that has been accepted for publication. As a service to our customers we are providing this early version of the manuscript. The manuscript will undergo copyediting, typesetting, and review of the resulting proof before it is published in its final citable form. Please note that during the production process errors may be discovered which could affect the content, and all legal disclaimers that apply to the journal pertain. 
95117, 95120, 95125, and 95165. We cross-referenced confirmed EoE patients and those treated with SCIT to identify EoE+SCIT and EoE-SCIT subjects, and extracted relevant data from the medical record (Supplemental Methods).

SCIT was based on percutaneous testing or ImmunoCAP allergen-specific IgE measurement. All allergen extracts from Greer Laboratories (Lenoir, NC). Histologic response to treatment was defined as a peak esophageal eosinophil count $<15$ eos/hpf. ${ }^{1,9}$ Symptom response was defined as the clinically reported patient global response (yes/no). We compared clinical, endoscopic, and histologic characteristics between EoE+SCIT and EoE-SCIT (Supplemental Methods).

Of 692 patients identified with EoE, 25 (3.6\%) had CPT codes for SCIT. Of those 25, $15 \mathrm{did}$ not meet inclusion (Supplemental Figure 1). When comparing the EoE-SCIT ( $n=667$ ) and EoE+SCIT ( $n=10)$ groups, age, sex, race, and baseline endoscopic and histologic features were similar (Table 1). The symptom duration prior to diagnosis of EoE was shorter in EoESCIT as compared to EoE+SCIT cohort (7.3 vs. 13.8 years; $\mathrm{p}=0.046$ ). As expected, the EoE +SCIT group had a higher rate of atopic disease.

For EoE+SCIT patients, immunotherapy dose remained consistent throughout the treatment course. The SCIT administered included dust mites, cat, dog, cockroach, tree, grass, weed, ragweed, English plantain, and Alternaria (Supplemental Table 1). Of the 10 cases, 9 were diagnosed with EoE before the administration of SCIT and none of the patients had started SCIT by the time of their first follow-up endoscopy after diagnosis, so we were able to assess initial treatment response in the absence of SCIT. Median follow-up time was 400 days.

After initial diagnosis, these 10 patients were treated with a topical steroid. At the first follow-up endoscopy, 3 (30\%) had histological response and $6(60 \%)$ had a symptom response. Of the 5 patients who had follow-up data and were concurrently treated with SCIT, $40 \%$ had a histological response and $40 \%$ had a symptom response. This did not vary by season of follow-up. These 5 patients had 20\% histological response and $40 \%$ symptomatic response after initial treatment prior to SCIT. Compared to the initial EGD, at follow up in the EoE+SCIT group there was less use of topical steroids ( $80 \%$ vs. $40 \%$ ) and more use of dietary elimination ( $0 \%$ vs. $80 \%$ ) (Supplemental Table 2). There were no adverse outcomes identified in the EoE+SCIT cohort.

The data from this study merit discussion. There were few differences between the EoE +SCIT vs EoE-SCIT groups, and due to a small number of patients treated with SCIT, we are unable to make firm conclusions regarding the impact on treatment outcomes. However, in this selected population there was no decrement in histologic response rates or worsening of global symptom responses in the EoE+SCIT cohort.

There is little literature exploring safety and efficacy of SCIT for EoE treatment. ${ }^{4-8}$ Ramirez et al describe a 4-year-old boy with refractory EoE who responded histologically and symptomatically to SCIT to dust mites. ${ }^{5}$ De Swert et al describe a 10-year-old boy who had symptomatic and histologic improvement after desensitization with SCIT to grass and birch allergens. ${ }^{4}$ Castilano et al describe a 30-year-old male who had resolution of symptoms and 
normalization of esophageal biopsies after SCIT to dust mites, cockroaches, weeds and trees. ${ }^{7}$ Notably, there is one case reporting exacerbation of symptoms after SCIT to grass pollen, implicating a potential risk in treatment of EoE with SCIT. ${ }^{8}$

Despite our review of a large database of EoE patients and a large data warehouse, we could identify only a small fraction of EoE patients who underwent treatment with SCIT at our center, with even fewer receiving SCIT after the initial diagnosis of EoE. Consequently, we are unable to make definitive conclusions regarding the role of SCIT. The number of cases were limited by a single center study, patients could have been seen by an outside allergy practice to receive SCIT, and some had changes in concomitant EoE treatment that could impact response. Further investigation should include multiple medical centers and community-based practices, as well as limit other treatment changes. Nevertheless, because we only included EoE subjects who we could confirm received SCIT, our data are valid in this small group, and should form the basis for future prospective studies using validated EoE outcomes and standardized SCIT protocols.

In conclusion, with the exception of increased atopic disease and longer duration of symptoms, there were few differences between EoE+SCIT and EoE-SCIT patients. The EoE +SCIT group had relatively low initial treatment response rates prior to SCIT. At follow-up, response rates were slightly improved, but in the context of less topical steroids and more dietary elimination. We did not observe any worse outcomes among patients in the EoE +SCIT cohort, suggesting that SCIT in EoE patients may be safe and is worthy of future study.

\section{Supplementary Material}

Refer to Web version on PubMed Central for supplementary material.

\section{Funding Source:}

This study was funded in part by NIH award T32 DK007634 (SE; CCR). It also used services from the NC Translational and Clinical Sciences (NC TraCS) Institute, which is supported by the National Center for Advancing Translational Sciences (NCATS) through Grant Award Number UL1TR002489.

Potential competing interests: Dr. Dellon is a consultant for Adare, Alivio, Allakos, Banner, Calypso, Enumeral, EsoCap, GSK, Gossamer Bio, Receptos/Celegene, Regeneron, Robarts, and Shire, receives research funding from Allakos, Adare, GSK, Meritage, Miraca, Nutricia, Receptos/Celgene, Regeneron, and Shire, and has received an educational grant from Allakos, Banner, and Holoclara. None of the other authors report and potential conflicts of interest with this study.

\section{Abbreviations:}

EoE

SCIT

OIT

EoE-SCIT

EoE+SCIT eosinophilic esophagitis

subcutaneous immunotherapy

oral immunotherapy

eosinophilic esophagitis without subcutaneous immunotherapy

eosinophilic esophagitis with subcutaneous immunotherapy 
ICD international classification of diseases

UNC University of North Carolina

\section{References}

1. Dellon ES, Gonsalves N, Hirano I, Furuta GT, Liacouras C, Katzka DA. ACG Clinical Guideline: Evidence based approach to the diagnosis and management of esophageal eosinophilia and eosinophilic esophagitis. Am J Gastroenterol. 2013;108(5):679-692. [PubMed: 23567357]

2. Pesek RD, Rettiganti M, O'Brien E, et al. Effects of allergen sensitization on response to therapy in children with eosinophilic esophagitis. Ann Allergy Asthma Immunol. 8 2017;119(2): 177-183. [PubMed: 28676207]

3. Wolf WA, Jerath MR, Dellon ES. De-novo onset of eosinophilic esophagitis after large volume allergen exposures. J Gastrointestin Liver Dis. 6 2013;22(2):205-208. [PubMed: 23799220]

4. De Swert L, Veereman G, Bublin M, et al. Eosinophilic gastrointestinal disease suggestive of pathogenesis-related class 10 (PR-10) protein allergy resolved after immunotherapy. J Allergy Clin Immunol. 2 2013;131(2):600-602 e601-603. [PubMed: 23374273]

5. Ramirez RM, Jacobs RL. Eosinophilic esophagitis treated with immunotherapy to dust mites. J Allergy Clin Immunol. 610 2013;132(2):503-504. [PubMed: 23763975]

6. Calderon MA. Could allergen immunotherapy be a therapeutic intervention in eosinophilic oesophagitis? J Allergy Clin Immunol. 2016;137 (Suppl 2):AB98.

7. Castilano A, Zacharias D. Immunotherapy as treatment for aeroallergen triggered eosinophilic esophagitis. Ann Allergy Asthma Immunol. 2013;111(5):A62.

8. Wells R, Fox AT, Furman M. Recurrence of eosinophilic oesophagitis with subcutaneous grass pollen immunotherapy. BMJ Case Rep. 315 2018;2018.

9. Runge TM, Eluri S, Woosley JT, Shaheen NJ, Dellon ES. Control of inflammation decreases the need for subsequent esophageal dilation in patients with eosinophilic esophagitis. Dis Esoph. 2017;30:1-7.

10. Rybnicek DA, Hathorn KE, Pfaff ER, Bulsiewicz WJ, Shaheen NJ, Dellon ES. Administrative coding is specific, but not sensitive, for identifying eosinophilic esophagitis.. Dis Esoph. 2014;27:703-708. 
Table 1.

Demographics and clinical characteristics of EoE patients with and without subcutaneous immunotherapy

\begin{tabular}{lccc}
\hline & EoE-SCIT $(\mathbf{n}=\mathbf{6 6 7})$ & EoE+SCIT (n = 10) & P value \\
\hline Age (mean years \pm SD) & $26.0 \pm 18.4$ & $29.2 \pm 14.2$ & 0.59 \\
Male (n, \%) & $459(69)$ & $5(50)$ & 0.20 \\
White (n, \%) & $532(81)$ & $9(90)$ & 0.46 \\
Symptoms (n, \%) & & & \\
$\quad$ Dysphagia & $466(71)$ & $8(80)$ & 0.52 \\
Heartburn & $249(39)$ & $4(40)$ & 0.94 \\
$\quad$ Abdominal pain & $143(22)$ & $1(10)$ & 0.36 \\
Symptom length prior to diagnosis (mean years \pm SD) & $7.3 \pm 8.6$ & $13.8 \pm 8.8$ & 0.046 \\
Atopy (n, \%) & & & \\
$\quad$ Rhinitis/sinusitis & $253(40)$ & $10(100)$ & 0.01 \\
$\quad$ Asthma & $156(24)$ & $6(60)$ & 0.01 \\
Food allergy & $173(29)$ & $4(44)$ & 0.32 \\
Baseline EGD findings & & & \\
$\quad$ Rings & $298(45)$ & $4(40)$ & 0.75 \\
Stricture & $134(20)$ & $3(30)$ & 0.45 \\
Narrowing & $93(14)$ & $2(20)$ & 0.60 \\
Furrows & $376(57)$ & $5(50)$ & 0.67 \\
Exudates/white plaques & $225(34)$ & $7(70)$ & 0.02 \\
Edema/decreased vascularity & $199(30)$ & $4(40)$ & 0.50 \\
$\quad$ Dilation performed & $140(21)$ & $3(30)$ & 0.50 \\
Baseline eosinophil counts (mean eos/hpf \pm SD) & $65.2 \pm 44.7$ & $72.8 \pm 36.5$ & 0.62 \\
\hline
\end{tabular}

